\title{
Prevalencia de cardiopatía isquémica asintomática en pacientes con diabetes mellitus tipo 2
}

\section{Dulman O. Pineda-De Paz ${ }^{a}$, Mario R. Pineda-De Paz ${ }^{a}$, Yu L. Lee-Tsai ${ }^{a}$, Clara E. Chang ${ }^{\mathrm{b}}$, Lissette C. Torres-Salazar ${ }^{\mathrm{c}}$ y Luis C. Barrios-Lupitou ${ }^{\mathrm{d}, *}$}

\author{
a Departamento de Medicina Interna, Hospital General San Juan de Dios, Guatemala, Guatemala \\ b Unidad de Cardiología, Departamento de Medicina Interna, Hospital General San Juan de Dios, Guatemala, Guatemala \\ c Unidad de Endocrinología, Departamento de Medicina Interna, Hospital General San Juan de Dios, Guatemala, Guatemala \\ ${ }^{\mathrm{d}}$ Facultad de Ciencias Médicas, Universidad de San Carlos de Guatemala, Departamento de Medicina Interna, Hospital General \\ San Juan de Dios, Guatemala, Guatemala
}

Recibido el 10 de mayo de 2017; aceptado el 28 de agosto de 2017

Disponible en Internet el 1 de diciembre de 2017

\section{PALABRAS CLAVE \\ Diabetes mellitus; Isquemia miocárdica; Electrocardiografía; Prueba de esfuerzo; Dobutamina}

\begin{abstract}
Resumen
Introducción: La enfermedad cardiovascular es la causa principal de muerte en pacientes con diabetes mellitus. La prevalencia de cardiopatía isquémica asintomática es más alta en pacientes diabéticos que en no diabéticos y se asocia a peor pronóstico.

Objetivo: Identificar la prevalencia de cardiopatía isquémica asintomática en pacientes con diabetes mellitus tipo 2 en un hospital de tercer nivel de atención de Guatemala y analizar la posible asociación de dicha enfermedad con características epidemiológicas, clínicas y metabólicas.

Métodos: Estudio de corte transversal en el que se estudió una muestra de 92 pacientes diabéticos seleccionados de forma aleatoria simple. Se realizó electrocardiograma, que cuando fue negativo para isquemia ameritó prueba de esfuerzo, o de lo contrario, ecocardiograma de estrés con dobutamina.

Resultados: La edad media de los participantes fue de 57 años, 88\% de los cuales eran mujeres; la duración media de la diabetes fue 7 años. Se encontró cardiopatía isquémica asintomática en el $22,8 \%$ de los casos. No se hallaron posibles asociaciones entre cardiopatía isquémica asintomática y edad, sexo, enfermedad arterial periférica, índice de masa corporal, índice tobillo-brazo, hipertensión arterial, dislipidemia, tabaquismo activo, sedentarismo, sobrepeso/obesidad, alcoholismo, glucosa en ayunas, hemoglobina glicosilada, colesterol total, colesterol HDL, colesterol LDL, ácido úrico, creatinina, tasa de filtrado glomerular y microalbuminuria.
\end{abstract}

\footnotetext{
* Autor para correspondencia.

Correos electrónicos: carlos.barrios@hotmail.com, lupitou@gmail.com (L.C. Barrios-Lupitou).
} 


\section{KEYWORDS}

Diabetes mellitus; Myocardial ischaemia; Electrocardiograph; Stress test; Dobutamine
Conclusiones: La prevalencia de cardiopatía isquémica asintomática en la población estudiada con diabetes mellitus tipo 2 fue de $22,8 \%$. No se encontraron posibles asociaciones de cardiopatía isquémica asintomática con las variables estudiadas.

(c) 2017 Sociedad Colombiana de Cardiología y Cirugía Cardiovascular. Publicado por Elsevier España, S.L.U. Este es un artículo Open Access bajo la licencia CC BY-NC-ND (http:// creativecommons.org/licenses/by-nc-nd/4.0/).

Prevalence of asymptomatic ischaemic heart disease in patients with type 2 diabetes mellitus

\begin{abstract}
Introduction: Cardiovascular disease is the main cause of death in patients with diabetes mellitus. The prevalence of asymptomatic ischaemic heart disease is higher in diabetic patients than in non-diabetic ones, and is associated with a worse prognosis.

Objective: To determine the prevalence of asymptomatic ischaemic heart disease in patients with type 2 diabetes mellitus in a third level of care hospital in Guatemala, as well as to analyse the possible relationship of this disease with epidemiological, clinical, and metabolic characteristics.

Methods: A cross-sectional study was conducted on a sample of 92 randomly selected diabetic patients. An electrocardiogram was performed, which when it was negative for ischaemia, an exercise stress test or a dobutamine stress echocardiogram was performed.

Results: The mean age of the participants was 57 years, $88 \%$ of whom were women. The mean duration of the diabetes was 7 years. Asymptomatic ischaemic heart disease was found in $22.8 \%$ of case. No significant associations were found between ischaemic heart disease and age, gender, peripheral arterial disease, body mass index, ankle-brachial index, arterial hypertension, dyslipidaemia, active smoking, sedentarism, overweight/obesity, alcoholism, fasting glucose, glycosylated haemoglobin, total cholesterol, HDL-cholesterol, LDL-cholesterol, uric acid, creatinine, glomerular filtration rate, and urine microalbumin.

Conclusions: The prevalence of asymptomatic ischaemic heart disease was $22.8 \%$ in the population studied with type 2 diabetes mellitus. No significant associations were found between ischaemic heart disease and the variables studied.

(c) 2017 Sociedad Colombiana de Cardiología y Cirugía Cardiovascular. Published by Elsevier España, S.L.U. This is an open access article under the CC BY-NC-ND license (http:// creativecommons.org/licenses/by-nc-nd/4.0/).
\end{abstract}

\section{Introducción}

La diabetes mellitus es una de las enfermedades crónicas no transmisibles más prevalentes en el mundo y conlleva un riesgo aumentado de desarrollar complicaciones cardíacas a lo largo de su evolución ${ }^{1}$.

De acuerdo con datos de la Federación Internacional de Diabetes, la prevalencia global de esta condición en personas de 20 a 79 años para el 2013 era de $8,3 \%$, y se estima que para el 2035 será de $8,8 \%^{2}$. La incidencia en América aumenta en forma sostenida, tanto de la diabetes tipo 1 como de la 2, y se considera un problema de salud pública ${ }^{3}$, que en 2008 alcanzó una prevalencia de 348 millones y un estimado de 438 para el 2030. Según datos de la misma entidad, la prevalencia de diabetes para Centroamérica y Sudamérica es del $8,0 \%{ }^{4}$.

Según diversos estudios, la enfermedad cardiovascular es la causa principal de muerte en pacientes con diabetes ${ }^{5,6}$; esta última se considera un factor de riesgo independiente para enfermedad cardiovascular y muerte ${ }^{7,8}$.

Existen numerosos estudios sobre el riesgo cardiovascular que representa la diabetes; en uno de los más representativos, el INTERHEART, presentó un riesgo atribuible de $9,9 \%{ }^{9}$.
De otro lado, la cardiopatía isquémica asintomática fue descrita hace aproximadamente medio siglo y desde entonces es motivo de múltiples investigaciones que han llevado al conocimiento de su significancia clínica, que además ha quedado bien establecida. Se define por hallazgos objetivos de isquemia miocárdica en ausencia de angina o equivalentes anginosos. Su prevalencia es muy variable, depende del método diagnóstico, y va desde $6,4 \%$ hasta $59 \%{ }^{10-13} ; 2$ a 7 veces más alta en diabéticos que en no diabéticos, y se asocia con un incremento de casi tres veces más de eventos cardiovasculares (HR 2,79) y peor pronóstico ${ }^{11}$.

Barthelemy et al. ${ }^{14}$ estudiaron 122 pacientes, $16 \%$ (22 casos) de los cuales tenían cardiopatía isquémica y $6 \%$ enfermedad arterial coronaria significativa.

Muchos diabéticos viven con lesiones coronarias significativas sin experimentar síntomas o signos de enfermedad arterial coronaria, principalmente angina. Aunque aún no se puede asegurar el mecanismo exacto de la isquemia miocárdica asintomática, se han propuesto muchos para explicarla y se cree que varios de ellos coexisten. De igual forma, se postula que los pacientes con cardiopatía isquémica asintomática tienen un umbral más alto para el dolor, al igual que se ha mencionado la existencia de un procesamiento 
anormal a nivel central de las señales aferentes cardíacas que llegan a través de fibras que transmiten el dolor. Otro mecanismo propuesto es la secreción de endorfinas (sustancias similares a los opioides) en respuesta al dolor de origen cardíaco, que aunque muy sugestivo, aún no es concluyente $\mathrm{e}^{10-13}$.

También se menciona el papel que desempeñan en la cardiopatía isquémica asintomática la neuropatía y la disfunción autonómica que desarrollan los pacientes diabéticos a lo largo de su evolución. De otro lado, estudios de isquemia mediante monitorización Holter revelan que el $80 \%$ de los episodios de isquemia ocurren de manera silente $e^{10-13}$.

Para la salud pública en general es importante identificar no sólo la prevalencia de esta entidad que está relacionada con un peor pronóstico, sino analizar los factores de riesgo asociados y establecer los subgrupos de alto y muy alto riesgo para cardiopatía isquémica asintomática, a fin de establecer un diagnóstico precoz e instaurar una estrategia temprana que evite eventos cardiovasculares mayores, así como para adoptar medidas de prevención oportunas en aquellos pacientes diabéticos que no han desarrollado cardiopatía isquémica asintomática. Por tanto, el objetivo principal de esta investigación fue identificar la prevalencia de cardiopatía isquémica asintomática en pacientes con diabetes mellitus tipo 2 en un hospital de tercer nivel de Guatemala y analizar su posible asociación con características epidemiológicas, clínicas y metabólicas.

\section{Métodos}

\section{Diseño y criterios de inclusión y exclusión}

Se llevó a cabo un estudio de corte transversal en 92 pacientes con diagnóstico de diabetes mellitus tipo 2 , de 18 a 75 años de edad, seleccionados en forma aleatoria simple, atendidos en la clínica de diabetes e hipertensión del Hospital General San Juan de Dios de la ciudad de Guatemala, institución de tercer nivel de atención y de referencia nacional.

Fueron criterios de exclusión: síntomas de cardiopatía isquémica; incapacidad de colaborar, participar y cumplir las pruebas requeridas en el estudio; antecedentes de eventos isquémicos coronarios previos o ingresos hospitalarios por dichas causas; solicitud propia de retirarse del estudio por alguna situación personal; enfermedad neurológica significativa que pudiera afectar la cognición; desorden psiquiátrico clínicamente significativo que pudiera afectar la capacidad del paciente para participar en el estudio; diagnóstico de aneurisma o disección aórtica; historia de enfermedad reumática o enfermedad inmunológica sistémica; embarazo y otras afecciones que incapacitaran al paciente para participar en el estudio durante el desarrollo del mismo o que fueran consideradas por los autores.

\section{Procedimientos}

Se registró edad, sexo, etnia, procedencia, escolaridad, antecedentes patológicos personales y familiares, duración de la diabetes en años, medicamentos que consumía, tabaquismo, ingesta de alcohol y actividad física. Los pacientes fueron evaluados clínicamente por peso, talla, índice de masa corporal, índice tobillo-brazo y presión arterial. En los expedientes clínicos se revisaron datos de los laboratorios más recientes: glucemia en ayunas, hemoglobina glicosilada, colesterol total, triglicéridos, colesterol LDL, colesterol HDL, creatinina y ácido úrico. Se realizó la prueba de microalbuminuria con tira reactiva de Micral Test $^{T M}$.

Para etnia se preguntó si se identificaban como mayas o no mayas. El índice tobillo-brazo se calculó dividiendo la presión sistólica del tobillo entre la del brazo, obtenida con la ayuda de un doppler arterial periférico y se consideró positiva si el índice era $<0,9$ y negativa si era $\geq$ 0,9 . Para consumo de alcohol se preguntó si el paciente refería consumir más de 10 gramos de alcohol al día en el caso de las mujeres y más de 20 gramos de alcohol al día en el caso de los hombres o si había consumido $\geq 4$ $0 \geq 5$ bebidas en un día en el último mes para mujeres y hombres respectivamente. Para tabaquismo se preguntó por número de cigarrillos consumidos por día. Para actividad física se registró el número de minutos de ejercicio por semana. El índice de masa corporal (IMC) se calculó de acuerdo con la fórmula IMC = peso en kilogramos/talla en metros elevado al cuadrado. Se consideró hipertensión arterial si refería uso de medicamentos antihipertensivos o si durante la evaluación física se encontró presión sistólica $\geq 140 \mathrm{~mm} \mathrm{Hg}$ y/o presión diastólica $\geq 90 \mathrm{~mm} \mathrm{Hg}$ después de 30 min en reposo en dos momentos distintos; se tuvo en cuenta el valor más alto.

Se consideró como dislipidemia la alteración de uno o más de los componentes lipídicos: triglicéridos $\geq 150 \mathrm{mg} / \mathrm{dl}$, colesterol total $\geq 200 \mathrm{mg} / \mathrm{dl}$, colesterol LDL mayor a $70 \mathrm{mg} / \mathrm{dl}$, por estar por encima de la meta para pacientes diabéticos, y colesterol $\mathrm{HDL} \leq 50 \mathrm{mg} / \mathrm{dl}$ y $\leq 40 \mathrm{mg} / \mathrm{dl}$ para hombres y mujeres respectivamente o que tomaran alguna estatina. Para la evaluación de la función renal se calculó la tasa de filtrado glomerular por la fórmula de Cockroft-Gault. El nivel de microalbuminuria fue medido con tira reactiva Micral Test ${ }^{T M}$; se consideró positivo si el resultado era $\geq$ $30 \mathrm{mg} / \mathrm{L}$.

Se tuvieron en cuenta signos sugestivos de cardiopatía isquémica en el electrocardiograma en reposo si se encontraban cambios del segmento ST y onda T (ondas T aplanadas o invertidas de ramas simétricas) con presencia de ondas Q anormales. Se consideró como prueba de esfuerzo positiva cuando se observó infradesnivel del segmento ST relativamente plano o descendente de $0,10 \mathrm{mV}(1 \mathrm{~mm})$, o más en relación con la unión $\mathrm{PQ}$, a más de $80 \mathrm{~ms}$ tras el punto $\mathrm{J}$ en tres latidos consecutivos (ST 80). En caso que el paciente tuviera frecuencia cardíaca mayor a 130 latidos/minuto, se tuvo en cuenta el infradesnivel del ST a partir de $60 \mathrm{~ms}$ tras el punto J (ST 60). El ecocardiograma de estrés con dobutamina se reportó positivo para cardiopatía isquémica cuando se observó alteración en la movilidad miocárdica segmentaria (hipocinesia/acinesia) vista en reposo y en estrés (infarto) o solo en estrés con movilidad normal en reposo (isquemia), asociada o no a cambios eléctricos.

Para la participación en el estudio se explicó a cada paciente en qué consistía la investigación, luego de lo cual cada uno leyó y firmó el consentimiento informado para poder ser incluido. Se contó con aprobación del Comité de Ética del Hospital. 


\section{Análisis estadísticos}

Para el cálculo de la muestra se estimó una prevalencia de cardiopatía isquémica asintomática del $6 \%$ reportada en estudios previos ${ }^{12}$, un nivel de confianza del $95 \%$, una precisión del $5 \%$ y un agregado del $10 \%$ por posibles pérdidas. Se tomó una muestra de 92 pacientes, de los que el $100 \%$ completó su participación en el estudio, de modo que no hubo excluidos. Se hizo una selección aleatoria simple con base en la lista de números aleatorios generados en línea en OpenEpi (www.openepi.com).

Los datos fueron ingresados y analizados en el programa Epi Info ${ }^{T M}$ 3.5.4. Se aplicó estadística descriptiva; en la comparación de grupos con cardiopatía isquémica asintomática y sin ésta, para las variables cuantitativas se aplicó t de Student no pareada y para variables cualitativas se calculó chi cuadrado; se consideraron diferencias estadísticamente significativas si $p<0,05$.

\section{Resultados}

La edad promedio de los participantes fue de $57 \pm 9$ años, $88 \%(n=81)$ mujeres y $98,8 \%(n=91)$ se identificó como no maya. El $71 \%(n=66)$ procedía de la ciudad capital. La duración promedio de la diabetes fue de $7 \pm 5$ años. El $6,5 \%(n=6)$ presentó enfermedad arterial periférica por un índice tobillo-brazo $<0,9$. El 21,7\% $(n=20)$ refirió antecedente familiar de enfermedad arterial coronaria. Para el tratamiento de la diabetes, $79,3 \%(n=73)$ refirió uso de antidiabéticos y $5,4 \%(n=5)$ de insulina glargina no NPH. La media de los factores de riesgo cardiovascular asociados a la diabetes (dislipidemia, hipertensión arterial, obesidad, sedentarismo y tabaquismo) fue de 2,86 $\pm 1,06$ (tabla 1).

En los resultados de los exámenes de laboratorios realizados se encontró que el promedio de hemoglobina glicosilada estaba por encima del valor que indica control de la enfermedad, el promedio de triglicéridos se encontró en límite máximo normal y el $68,4 \%$ presentó microalbuminuria (tabla 2).

Se diagnosticó cardiopatía isquémica asintomática en el $22,8 \%$ (IC 95\% 14,7\% - 32,7\%) $(\mathrm{n}=21)$ de los pacientes; 3 mediante electrocardiograma en reposo, 13 por prueba de esfuerzo y 5 por ecocardiograma de estrés con dobutamina (fig. 1).

En los pacientes sin cardiopatía isquémica asintomática se halló un promedio de triglicéridos más alto que el de los pacientes con ésta $(p=0,029)$. Los pacientes con cardiopatía isquémica asintomática tenían en promedio más edad y tasa de filtración glomerular, fue más frecuente la enfermedad arterial periférica, el tabaquismo activo, el sedentarismo y el alcoholismo, pero fue menor el promedio de tiempo de padecer diabetes, índice de masa corporal, índice tobillo/brazo, factores de riesgo cardiovascular, glucosa en ayunas, hemoglobina glicosilada, colesterol total, colesterol LDL, ácido úrico, creatinina, hipertensión arterial, dislipidemia y sobrepeso/obesidad, aunque no se encontraron diferencias significativas (tabla 3).

\section{Discusión}

En este estudio la prevalencia de cardiopatía isquémica asintomática en diabéticos fue de $22,8 \%$. Los pacientes sin este
Tabla 1 Características generales

\begin{tabular}{|c|c|}
\hline Características & $\mathrm{n}=92(\%)$ \\
\hline Edad promedio en años (DE*) & $57(9)$ \\
\hline Mujeres & $81(88)$ \\
\hline Etnia no maya & $91(98,8)$ \\
\hline Procedencia de la capital & $66(71,7)$ \\
\hline $\begin{array}{l}\text { Duración promedio de la diabetes, } \\
\left.\text { años ( } \mathrm{DE}^{*}\right)\end{array}$ & $7(5)$ \\
\hline Enfermedad arterial periférica & $6(6,5)$ \\
\hline $\begin{array}{l}\text { Historia familiar de enfermedad } \\
\text { coronaria }\end{array}$ & $20(21,7)$ \\
\hline $\begin{array}{l}\text { Índice de masa corporal promedio, } \\
\mathrm{kg} / \mathrm{m}^{2}\left(\mathrm{DE}^{*}\right)\end{array}$ & $28,6(4,9)$ \\
\hline $\begin{array}{l}\text { Presión arterial sistólica promedio, } \\
\mathrm{mm} \mathrm{Hg} \mathrm{(DE*)}\end{array}$ & $130(21)$ \\
\hline $\begin{array}{l}\text { Presión arterial diastólica promedio, } \\
\text { mm } \mathrm{Hg}\left(\mathrm{DE}^{*}\right)\end{array}$ & $75(11)$ \\
\hline Hipertensión arterial & $61(66,3)$ \\
\hline Dislipidemia & $69(75)$ \\
\hline Tabaquismo activo & $5(5,4)$ \\
\hline Sedentarismo & $77(83,6)$ \\
\hline Obesidad & $34(36,9)$ \\
\hline Alcoholismo & $5(5,4)$ \\
\hline Tratamiento con antidiabéticos orales & $73(79,3)$ \\
\hline $\begin{array}{l}\text { Tratamiento con antidiabéticos orales } \\
\text { más insulina }\end{array}$ & $13(14,1)$ \\
\hline Tratamiento con insulina & $5(5,4)$ \\
\hline Promedio de factores de riesgo (DE $)$ & $2,86(1,06)$ \\
\hline
\end{tabular}

* DE: desviación estándar.

Tabla 2 Resultados de laboratorios

\begin{tabular}{|c|c|}
\hline Exámenes & \\
\hline $\begin{array}{l}\text { Glucosa en ayunas promedio, } \mathrm{mg} / \mathrm{dl} \\
\left(\mathrm{DE}^{*}\right)\end{array}$ & $152(60)$ \\
\hline $\begin{array}{l}\text { Hemoglobina glicosilada promedio, } \\
\%\left(\mathrm{DE}^{*}\right)\end{array}$ & $8,4(2,2)$ \\
\hline $\begin{array}{l}\text { Colesterol total promedio, } \mathrm{mg} / \mathrm{dl} \\
\left(\mathrm{DE}^{*}\right)\end{array}$ & $208(48)$ \\
\hline $\begin{array}{l}\text { Colesterol HDL promedio, } \mathrm{mg} / \mathrm{dl} \\
\text { (DE*) }\end{array}$ & $41,8(9,3)$ \\
\hline $\begin{array}{l}\text { Colesterol LDL promedio, } \mathrm{mg} / \mathrm{dl} \\
\left(\mathrm{DE}^{*}\right)\end{array}$ & $124,7(38,5)$ \\
\hline Triglicéridos promedio, mg/dl (DE*) & $200(75)$ \\
\hline Ácido úrico promedio, mg/dl (DE*) & $6(1,9)$ \\
\hline Creatinina promedio, $\mathrm{mg} / \mathrm{dl}\left(\mathrm{DE}^{*}\right)$ & $0,88(0,33)$ \\
\hline $\begin{array}{l}\text { TFG }^{a} \text { por Cockcroft Gault promedio, } \\
\mathrm{ml} / \mathrm{min}\left(\mathrm{DE}^{*}\right)\end{array}$ & $82(27)$ \\
\hline Presencia de microalbuminuria (\%) & $63(68,4)$ \\
\hline
\end{tabular}

tipo de cardiopatía tuvieron valores de triglicéridos más altos que aquellos con cardiopatía isquémica asintomática. La prevalencia de cardiopatía isquémica asintomática se ha evaluado en múltiples estudios, en los que la reportan de dos a siete veces más alta en diabéticos que en no diabéticos y la asocian con un pronóstico pobre ${ }^{15,16}$. La importancia de la enfermedad cardiovascular radica en que, de acuerdo 


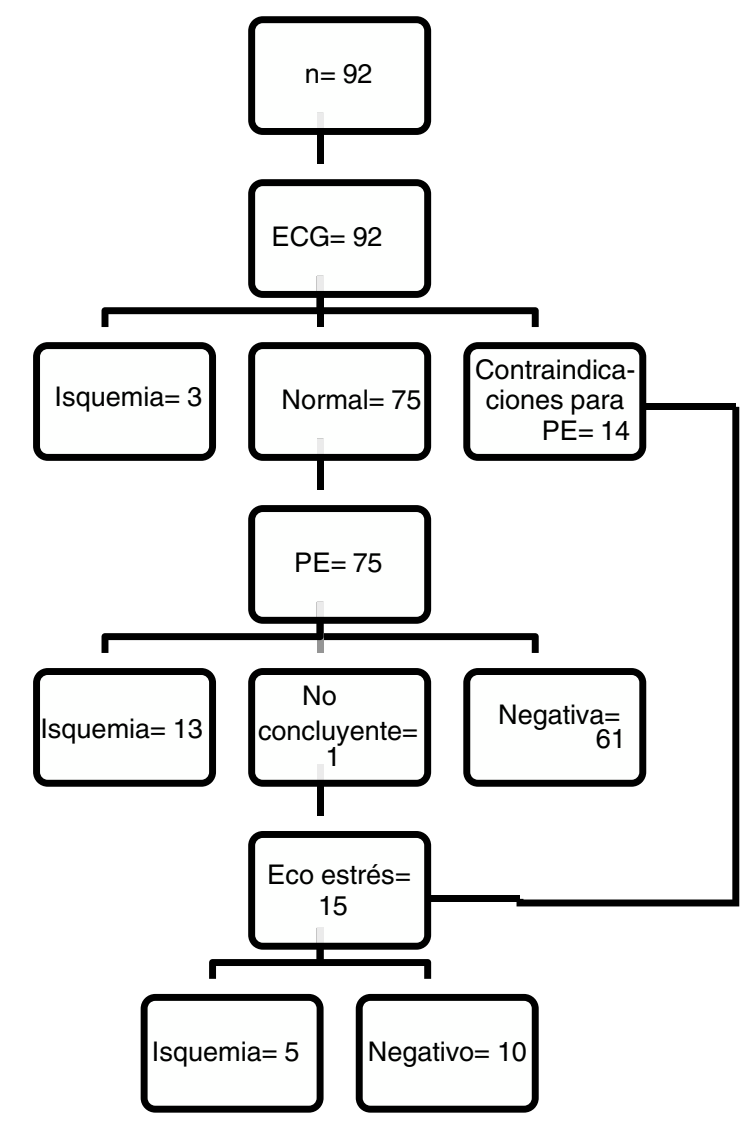

Figura 1 Estudios realizados para el diagnóstico de CIA.

con diferentes estudios, es la principal causa de muerte en pacientes con diabetes ${ }^{17-19}$, aunado a que la mortalidad en diabéticos que han tenido un infarto previo es más alta que en pacientes no diabéticos y mayor aún a pesar de haber sido revascularizados ${ }^{20-22}$. Por ende, es trascendental identificar el problema cardíaco silencioso en este grupo de pacientes, a fin de mejorar el pronóstico a través de la toma de medidas correctivas.

La prevalencia de cardiopatía isquémica asintomática encontrada en esta investigación no difiere considerablemente de la reportada en otros estudios, sin embargo existe un rango muy amplio el cual varía desde $6 \%$ hasta $59 \%$, diferencias que pueden obedecer a la selección de los participantes, así como a los métodos diagnósticos empleados ${ }^{23-29}$. El impacto de estas cifras indica la necesidad de un mejor control de los pacientes con diabetes para prevenir esta complicación e intervenir de forma adecuada a aquellos que ya la padecen. Al respecto, se conoce también que la cardiopatía isquémica asintomática tiene un alto valor pronóstico de eventos coronarios ${ }^{30,31}$.

Por otra parte, suscita controversia a quiénes y qué pruebas diagnósticas hacer; en general se recomienda realizarlas sólo en casos con riesgo moderado o alto de enfermedad cardiovascular ${ }^{32-36}$.

Así mismo, se discuten los métodos diagnósticos a emplear debido al alto costo de algunos de ellos $^{37}$; en este estudio, además del electrocardiograma y de la prueba de esfuerzo, se usó la prueba de estrés con dobutamina, considerada una buena alternativa, de bajo costo y mayor sensibilidad y especificidad, frente a la cardiología nuclear ${ }^{38}$ que puede tener mejor sensibilidad y especificidad pero costos más elevados; no obstante, con ello no se quiere decir que deban ser desplazados.

En diversos estudios se ha demostrado la asociación de los lípidos con la enfermedad isquémica, incluso como predictores de dicha enfermedad ${ }^{39-42}$. En este estudio, en los pacientes sin cardiopatía isquémica asintomática los niveles de triglicéridos fueron más altos que en aquellos con ésta $(p=0,029)$. En otros estudios no se ha encontrado esta asociación ${ }^{43}$, o al contrario, los pacientes con cardiopatía isquémica asintomática han tenido niveles de triglicéridos más elevados. Son varios los factores que pueden modificar los valores de triglicéridos, que pudieran explicar los resultados en este estudio, pero que no se tuvieron en cuenta durante la aleatorización; por ejemplo: grupos de medicamentos que estuvieran tomando algunos pacientes, respecto a lo que está bien establecido que pueden modificar de alguna manera los niveles de triglicéridos ${ }^{44,45}$. En primer lugar, las estatinas pueden modificar los niveles de triglicéridos reduciéndolos desde un 10 a un $20 \%$ y aún más (22 - 45\%) si los valores iniciales de triglicéridos son más altos; los fibratos que pueden reducir los niveles de triglicéridos y los antihipertensivos que pueden interactuar con el metabolismo de las lipoproteínas (estímulo Beta 1), principalmente los betabloqueadores cardioselectivos sin efectos beta 2 agonistas, e incrementar los triglicéridos.

En esta investigación no se encontró asociación significativa de ninguna otra característica con cardiopatía isquémica asintomática. Posiblemente el tamaño de la muestra no permitió encontrar asociaciones entre cardiopatía isquémica asintomática y las características de los pacientes estudiados sin que ello signifique que no existan; sin embargo debe mencionarse el porcentaje elevado de microalbuminuria de ambos grupos $(68,4 \%)$, que aunque no resultó estadísticamente significativo en este estudio, en otros la asociación de microalbuminuria y cardiopatía isquémica asintomática representa un grupo particularmente de alto riesgo para futuros eventos cardiovasculares.

\section{Limitaciones}

Sólo se incluyeron pacientes de las consultas externas de diabetes e hipertensión, por lo cual es representativo únicamente de esta población y no de toda la población guatemalteca; sin embargo, por razones sociales esta es la característica en estos centros de atención, en los que la mayoría de pacientes que asisten a sus consultas son mujeres. Aunque el sexo masculino es un factor de riesgo cardiovascular para enfermedad arterial coronaria, la postmenopausia también lo es y en este estudio la mayoría de mujeres estaba en edad posmenopáusica. Así mismo, para el estudio de isquemia se les realizó la prueba de esfuerzo o ecocardiograma estrés con dobutamina, que si bien existen pruebas disponibles con mayor sensibilidad y especificidad como la tomografía por emisión de fotón simple (SPECT) con tecnecio 99 o talio 201, hasta el día de hoy son inaccesibles en muchas ocasiones por su alto costo. 
Tabla 3 Características generales y cardiopatía isquémica asintomática

\begin{tabular}{|c|c|c|c|}
\hline & $\begin{array}{l}\text { Cardiopatía } \\
\mathrm{n}=21 \text { (\%) }\end{array}$ & $\begin{array}{l}\text { No cardiopatía } \\
\mathrm{n}=71(\%)\end{array}$ & Valor $\mathrm{p}$ \\
\hline Edad promedio en años (DE') & $59,7(10,1)$ & $56,7(9,6)$ & 0,226 \\
\hline \multicolumn{4}{|l|}{ Sexo } \\
\hline Femenino & $18(22,2)$ & $63(77,7)$ & \multirow[t]{2}{*}{0,709} \\
\hline Masculino & $3(27,2)$ & $8(72,7)$ & \\
\hline Duración promedio de la diabetes, años (DE) & $7,2(5,5)$ & $7,4(5,7)$ & 0,977 \\
\hline Enfermedad arterial periférica & $2(9,52)$ & $4(5,63)$ & 0,616 \\
\hline Índice de masa corpora promedio, $\mathrm{kg} / \mathrm{m}^{2}(D E)$ & $28,05(5,37)$ & $28,85(4,81)$ & 0,526 \\
\hline Índice tobillo brazo, promedio (DE') & $1,04(0,10)$ & $1,05(0,12)$ & 0,835 \\
\hline Hipertensión arterial & $13(61,90)$ & $48(67,61)$ & 0,611 \\
\hline Dislipidemia & $14(66,67)$ & $55(77,46)$ & 0,390 \\
\hline Tabaquismo activo & $3(14,29)$ & $5(7,04)$ & 0,376 \\
\hline Sedentarismo & $20(95,24)$ & $57(80,28)$ & 0,176 \\
\hline Sobrepeso/Obesidad & $14(66,67)$ & $57(80,28)$ & 0,515 \\
\hline Alcoholismo & $2(9,52)$ & $3(4,23)$ & 0,320 \\
\hline Factores de riesgo, promedio (DE*) & $2,85(1,01)$ & $2,87(1,08)$ & 0,951 \\
\hline Glucosa en ayunas, $\mathrm{mg} / \mathrm{dl}\left(D E^{\prime}\right)$ & $145,80(54,03)$ & $154,97(62,74)$ & 0,546 \\
\hline Hemoglobina glicosilada, \% (DE') & $8,02(1,60)$ & $8,58(2,44)$ & 0,765 \\
\hline Colesterol total, mg/dl (DE') & $194(48,46)$ & $213,12(47,21)$ & 0,109 \\
\hline Colesterol HDL, mg/dl (DE*) & $43,47(7,80)$ & $41,36(9,79)$ & 0,367 \\
\hline Colesterol LDL, mg/dl (DE') & $115,90(38,10)$ & $127,38(38,56)$ & 0,232 \\
\hline Triglicéridos, mg/dl (DE') & $169,28(65,36)$ & $209,77(75,94)$ & 0,029 \\
\hline Ácido úrico, mg/dl (DE*) & $6,00(1,74)$ & $6,01(1,99)$ & 0,982 \\
\hline Creatinina, mg/dl (DE') & $0,85(0,23)$ & $0,89(0,35)$ & 0,774 \\
\hline TFG ${ }^{\mathrm{a}}$ por Cockcroft Gault, $\mathrm{ml} / \mathrm{min}\left(\mathrm{DE}^{*}\right)$ & $83,95(36,10)$ & $81,49(25,29)$ & 0,776 \\
\hline Microalbuminuria & $16(76,19)$ & $47(66,20)$ & 0,436 \\
\hline
\end{tabular}

* DE: desviación estándar

a TFG: tasa de filtrado glomerular.

\section{Conclusiones}

En este estudio de pacientes diabéticos asintomáticos atendidos en un centro de referencia en Guatemala, la prevalencia de cardiopatía isquémica asintomática fue del $22,8 \%$. Probablemente, debido al tamaño reducido de la muestra no se logró encontrar posibles asociaciones entre cardiopatía isquémica asintomática y las características sociodemográficas, clínicas y metabólicas de los pacientes incluidos en el estudio. Es necesario iniciar un estudio longitudinal que incluya mayor número de pacientes diabéticos asintomáticos, en el que se utilice un solo medio diagnóstico no invasivo con mayor sensibilidad y especificidad como la tomografía computarizada por emisión de fotón único (SPECT) con tecnecio 99. De igual forma, convendría establecer las características de los grupos de alto y muy alto riesgo de presentar eventos cardiovasculares mayores $\mathrm{y}$ hacer seguimiento a largo plazo para evidenciar los beneficios del diagnóstico precoz de la enfermedad arterial coronaria significativa asintomática y de las estrategias de revascularización temprana en aquellos pacientes que lo ameriten, con el objetivo de reducir el número de eventos coronarios agudos en este grupo de pacientes en alto riesgo y evolución desfavorable.

\section{Responsabilidades éticas}

Protección de personas y animales. Los autores declaran que para esta investigación no se han realizado experimentos en seres humanos ni en animales.

Confidencialidad de los datos. Los autores declaran que han seguido los protocolos de su centro de trabajo sobre la publicación de datos de pacientes.

Derecho a la privacidad y consentimiento informado. Los autores declaran que en este artículo no aparecen datos de pacientes.

\section{Conflicto de intereses}

Ninguno

\section{Bibliografía}

1. American Diabetes Association. Diagnosis and classification of diabetes mellitus. Diabetes Care. 2012;35 Suppl 1:S64-S71. doi: $10.2337 / \mathrm{dc} 12$-s064.

2. Guariguata L, Whiting DR, Hambleton I, Beagley J, Linnenkamp $U$, Shaw JE. Global estimates of diabetes 
prevalence for 2013 and projections for 2035. Diabetes Res Clin Pract. 2014;103:137-49, http://dx.doi.org/ 10.1016/j.diabres.2013.11.002

3. Barceló A, Rajpathak S. Incidence and prevalence of diabetes mellitus in the Americas. Rev Panam salud puíblica=Pan Am J public Heal. 2001;10:300-8, http://dx.doi.org/ 10.1590/S1020-49892001001100002

4. Aschner P, Aguilar-Salinas C, Aguirre L, Franco L, Gagliardino $\mathrm{J}$, Gorban S, et al. Diabetes in South and Central America: An update. Diabetes Res Clin Pract. 2014;103:238-43, http://dx.doi.org/10.1016/j.diabres.2013.11.010

5. Morrish NJ, Wang SL, Stevens LK, Fuller JH, Keen H. Mortality and causes of death in the WHO Multinational Study of Vascular Disease in Diabetes. Diabetologia. 2001:S14-21, http://dx.doi.org/10.1007/PL00002934, 44 Suppl 2(Suppl 2).

6. Malik S, Wong ND, Franklin SS, Kamath TV, L'Italien GJ, Pio Jr, et al. Impact of the metabolic syndrome on mortality from coronary heart disease, cardiovascular disease, and all causes in United States adults. Circulation. 2004;110:1245-50, http://dx.doi.org/10.1161/01.CIR. 0000140677.20606.0E

7. Franco OH, Steyerberg EW, Hu FB, Mackenbach J, Nusselder W. Associations of diabetes mellitus with total life expectancy and life expectancy with and without cardiovascular disease. Arch Intern Med. 2007;167:1145-51, http://dx.doi.org/10.1001/archinte.167.11.1145

8. Austin MA. Plasma triglyceride and coronary heart disease. Arterioscler Thromb. 1991;11:2-14, http://dx.doi.org/ 10.1161/01.ATV.11.1.2t

9. Yusuf S, Hawken S, Ounpuu S, Dans T, Avezum A, Lanas F, et al. Effect of potentially modifiable risk factors associated with myocardial infarction in 52 countries (the INTERHEART study): case-control study. Lancet. 2004;364:937-52, http://dx.doi.org/10.1016/S0140-6736(04)17018-9

10. Airaksinen KE. Silent coronary artery disease in diabetes-a feature of autonomic neuropathy or accelerated atherosclerosis? Diabetologia. 2001;44:259-66, http://dx.doi.org/10.1007/s001250051609

11. Blanchet Deverly A, Amara M, Larifla L, VelayoudomCéphise FL, Roques F, Kangambega $P$, et al. Silent myocardial ischaemia and risk factors in a diabetic AfroCaribbean population. Diabetes Metab. 2011;37:533-9, http://dx.doi.org/10.1016/j.diabet.2011.05.006

12. Milan Study on Atherosclerosis and Diabetes (MiSAD). Prevalence of Unrecognized Silent Myocardial Ischemia and Its Association With Atherosclerotic Risk Factors in NoninsulinDependent Diabetes Mellitus. Am J Cardiol. 1997; 79(2):134-39. doi:10.1016/S0002-9149(96)00699-6.

13. Miller TD, Rajagopalan N, Hodge DO, Frye RL, Gibbons RJ. Yield of stress single-photon emission computed tomography in asymptomatic patients with diabetes. Am Heart J. 2004;147:890-6, http://dx.doi.org/10.1016/j.ahj.2003.08.015

14. Barthelemy $O$, Jacqueminet $S$, Rouzet $F$, Isnard $R$, Bouzamondo A, Le Guludec D, et al. Intensive cardiovascular risk factors therapy and prevalence of silent myocardial ischaemia in patients with type 2 diabetes. Arch Cardiovasc Dis. 2008;101:539-46, http://dx.doi.org/10.1016/j.acvd.2008.06.015

15. IDF, Diabetes Atlas, Group. Update of mortality attributable to diabetes for the IDF Diabetes Atlas: Estimates for the year 2013. Diabetes Res Clin Pract. 2015;109:461-5, http://dx.doi.org/10.1016/j.diabres.2015.05.037

16. Valensi P, Pariès J, Brulport-Cerisier V, Torremocha F, Sachs RN, Vanzetto $G$, et al. Predictive value of silent myocardial ischemia for cardiac events in diabetic patients: influence of age in a French multicenter study. Diabetes Care. 2005;28:2722-7, http://dx.doi.org/10.2337/diacare.28.11.2722

17. De Backer G, Ambrosioni E, Borch-Johnsen K, Brotons C, Cifkova R, Dallongeville $\mathrm{J}$, et al. European guidelines on cardiovascular disease prevention in clinical practice: third joint task force of European and other societies on cardiovascular disease prevention in clinical practice (constituted by representatives of eight societies and by invit. Eur $\mathrm{J}$ Cardiovasc Prev Rehabil. 2003;10:S1-10, http://dx.doi.org/ 10.1097/01.hjr. 0000087913.96265.e2

18. Gu K, Cowie CC, Harris Ml. Mortality in adults with and without diabetes in a national cohort of the U.S. population, 1971-1993. Diabetes Care. 1998;21:1138-45, http://dx.doi.org/10.2337/diacare.21.7.1138

19. Kannel WB, McGee DL. Diabetes and cardiovascular disease. The Framingham study. JAMA. 1979;241:2035-8, http://dx.doi.org/10.1001/jama.1979.03290450033020

20. Cho E, Rimm EB, Stampfer MJ, Willett WC, Hu FB. The impact of diabetes mellitus and prior myocardial infarction on mortality from all causes and from coronary heart disease in men. J Am Coll Cardiol. 2002;40:954-60, http: //dx.doi.org/10.1016/S0735-1097(02)02044-2

21. Donnan PT, Boyle DIR, Broomhall J, Hunter K, MacDonald TM, Newton RW, et al. Prognosis following first acute myocardial infarction in Type 2 diabetes: a comparative population study. Diabet Med. 2002;19:448-55, http://dx.doi.org/ 10.1046/j.1464-5491.2002.00711.x

22. Haffner SM, Lehto S, Rönnemaa T, Pyörälä K, Laakso $M$. Mortality from coronary heart disease in subjects with type 2 diabetes and in nondiabetic subjects with and without prior myocardial infarction. N Engl J Med. 1998;339:229-34, http://dx.doi.org/10.1056/NEJM199807233390404

23. Beller GA. Noninvasive screening for coronary atherosclerosis and silent ischemia in asymptomatic type 2 diabetic patients: is it appropriate and cost-effective? J Am Coll Cardiol. 2007;49:1918-23, http://dx.doi.org/ 10.1016/j.jacc.2007.01.079

24. Davis TME, Coleman RL, Holman RR. Prognostic significance of silent myocardial infarction in newly diagnosed type 2 diabetes mellitus: United Kingdom prospective diabetes study (UKPDS) 79. Circulation. 2013;127:980-7, http: / /dx.doi.org/10.1161/CIRCULATIONAHA.112.000908

25. De Lorenzo A, Lima RS, Siqueira-Filho AG, Pantoja MR. Prevalence and prognostic value of perfusion defects detected by stress technetium-99m sestamibi myocardial perfusion single-photon emission computed tomography in asymptomatic patients with diabetes mellitus and no known coronary artery disease. Am J Cardiol. 2002;90:827-32, doi: S0002914902027029 [pii].

26. Hage FG, Lusa L, Dondi M, Giubbini R, Iskandrian AE. Exercise stress tests for detecting myocardial ischemia in asymptomatic patients with diabetes mellitus. Am J Cardiol. 2013;112:14-20, http://dx.doi.org/10.1016/j.amjcard.2013.02.047

27. Koistinen MJ. Prevalence of asymptomatic myocardial ischaemia in diabetic subjects. BMJ. 1990:92-5, http://dx.doi.org/10.1136/bmj.301.6743.92, 301(July).

28. MacDonald MR, Petrie MC, Home PD, Komajda M, Jones NP, Beck-Nielsen $\mathrm{H}$, et al. Incidence and prevalence of unrecognized myocardial infarction in people with diabetes: a substudy of the Rosiglitazone Evaluated for Cardiac Outcomes and Regulation of Glycemia in Diabetes (RECORD) study. Diabetes Care. 2011;34:1394-6, http://dx.doi.org/10.2337/dc10-2398

29. Wackers FJT, Young LH, Inzucchi SE, Chyun DA, Davey JA, Barrett EJ, et al. Detection of silent myocardial ischemia in asymptomatic diabetic subjects: The DIAD study. Diabetes Care. 2004;27:1954-61, http://dx.doi.org/ 10.2337/diacare.27.8.1954

30. American Diabetes Association. Consensus development conference on the diagnosis of coronary heart disease in people with diabetes: 10-11 February 1998, Miami, Florida. American Diabetes Association. Diabetes Care. 1998; 21(9):1551-59. doi:10.2337/diacare.21.9. 1551. 
31. Diamond GA, Kaul S, Shah PK. Screen Testing. Cardiovascular Prevention in Asymptomatic Diabetic Patients. J Am Coll Cardiol. 2007;49:1915-7, http://dx.doi.org/ 10.1016/j.jacc.2006.09.057

32. Bax JJ, Bonow RO, Tschöpe D, Inzucchi SE, Barrett E. Global Dialogue Group for the Evaluation of Cardiovascular Risk in Patients With Diabetes. The potential of myocardial perfusion scintigraphy for risk stratification of asymptomatic patients with type 2 diabetes. J Am Coll Cardiol. 2006;48:754-60, http://dx.doi.org/10.1016/j.jacc.2006.04.077

33. Janand-Delenne B, Savin B, Habib G, Bory M, Vague P, Lassmann-Vague V. Silent myocardial ischemia in patients with diabetes: who to screen. Diabetes Care. 1999;22:1396-400, http://dx.doi.org/10.2337/diacare.22.9.1396

34. Miller TD, Redberg RF, Wackers FJT. Screening asymptomatic diabetic patients for coronary artery disease: why not? J Am Coll Cardiol. 2006;48:761-4, http://dx.doi.org/ 10.1016/j.jacc.2006.04.076

35. Rutter MK, Nesto RW. The changing costs and benefits of screening for asymptomatic coronary heart disease in patients with diabetes. Nat Clin Pract Endocrinol Metab. 2007;3:26-35, http://dx.doi.org/10.1038/ncpendmet0352

36. Valensi P. Silent myocardial ischaemia. To screen or not to screen? That is. Arch Cardiovasc Dis. 2008;101:509-11, http://dx.doi.org/10.1016/j.acvd.2008.10.001

37. Heydari B, Juan Y, Liu H, Abbasi S, Shah R, Blankstein R, et al. Stress Perfusion Cardiac Magnetic Resonance Imaging Effectively Risk Stratifies Diabetic Patients With Suspected Myocardial Ischemia. Circ Cardiovasc Imaging. 2016;9:e004136, http://dx.doi.org/10.1161/CIRCIMAGING.115.004136

38. Penfornis A, Zimmermann C, Boumal D, Sabbaht A, Meneveau N, Gaultier-Bourgeois $S$, et al. Use of dobutamine stress echocardiography in detecting silent myocardial ischaemia in asymptomatic diabetic patients: A comparison with thallium scintigraphy and exercise testing. Diabet Med. 2001;18:900-5, http://dx.doi.org/10.1046/j.1464-5491.2001.00599.x

39. Castelli WP. Epidemiology of triglycerides: a view from Framingham. Am J Cardiol. 1992;70:3H-9H, http://dx.doi.org/10.1016/0002-9149(92)91083-G

40. Gotto AM. Triglyceride as a risk factor for coronary artery disease. Am J Cardiol. 1998;82:22-5, http://dx.doi.org/ 10.1016/S0002-9149(98)00770-X

41. Jeppesen J, Hein HO, Suadicani P, Gyntelberg F. Triglyceride concentration and ischemic heart disease: an eight-year follow-up in the Copenhagen Male Study. Circulation. 1998;97:1029-36, http://dx.doi.org/10.1161/ 01.CIR.97.11.1029

42. Yarnell JW, Patterson CC, Sweetnam PM, Thomas HF, Bainton D, Elwood PC, et al. Do total and high density lipoprotein cholesterol and triglycerides act independently in the prediction of ischemic heart disease? Ten-year follow-up of Caerphilly and Speedwell Cohorts. Arterioscler Thromb Vasc Biol. 2001;21:1340-5, http://dx.doi.org/10.1161/hq0801.093505

43. Tsai JC, Chang D, Chung F, Wu J, Shin S, Lee Y. The association of silent coronary artery disease and metabolic syndrome in Chinese with type 2 diabetes mellitus. Rev Diabet Stud. 2004;1:18-28, http://dx.doi.org/10.1900/RDS.2004.1.18

44. Huxley R, Barzi F, Woodward M. Excess risk of fatal coronary heart disease associated with diabetes in men and women: meta-analysis of 37 prospective cohort studies. BMJ. 2006;332:73-8, http://dx.doi.org/ 10.1136/bmj.38678.389583.7C

45. Peña Y, Fernandez-Britto JE, Bacallao J, Batista JF, de Leon $M L$. Lipid levels as predictors of silent myocardial ischemia in a type 2 diabetic population in Havana. MEDICC Rev. 2012;14: $18-24$. 